\title{
An Infrared Renormalization Group Limit Cycle in QCD
}

\author{
Eric Braaten \\ Department of Physics, The Ohio State University, Columbus, OH 43210, USA \\ H.-W. Hammer \\ Helmholtz-Institut für Strahlen- und Kernphysik (Abt. Theorie), Universität Bonn, 53115 Bonn, Germany
}

(Dated: March 17, 2003)

\begin{abstract}
Small increases in the up and down quark masses of QCD would tune the theory to the critical renormalization group trajectory for an infrared limit cycle in the three-nucleon system. At critical values of the quark masses, the deuteron binding energy goes to zero and the triton has infinitely many excited states with an accumulation point at the 3-nucleon threshold. The ratio of the binding energies of successive states approaches a universal constant that is close to 515 . The proximity of physical QCD to the critical trajectory for this limit cycle explains the success of an effective field theory of nucleons with contact interactions only in describing the low-energy 3-nucleon system.
\end{abstract}

PACS numbers: 12.38.Aw, 21.45.+v, 11.10.Hi, 64.60.Ak

Keywords: Renormalization group, limit cycle, quantum chromodynamics

The development of the renormalization group ( $R G)$ has had a profound effect on many branches of physics. Its successes range from explaining the universality of critical phenomena in condensed matter physics to the nonperturbative formulation of quantum field theories that describe elementary particles [1]. Most simple applications of the renormalization group involve fixed points that are approached either in the infrared limit or in the ultraviolet limit. However, the renormalization group can be reduced to a set of differential equations that define a flow in the space of coupling constants. A fixed point where the flow vanishes is only the simplest topological feature that can be exhibited by such a flow. Another topological feature is a limit cycle, which is a 1dimensional orbit that is closed under the RG flow. The possibility of RG flow to a limit cycle was proposed by Wilson in 1971 [2]. Glazek and Wilson have recently constructed a simple discrete Hamiltonian system that exhibits RG flow to a limit cycle [3]. However, few physical applications of RG limit cycles have emerged.

The purpose of this letter is to point out that Quantum Chromodynamics (QCD) is close to the critical trajectory for an infrared RG limit cycle in the 3-nucleon sector. It can be tuned to the critical trajectory by small changes in the up and down quark masses. The proximity of the physical quark masses to these critical values explains the success of a program initiated by Efimov to describe the 3 -nucleon problem in terms of zero-range forces between nucleons [4]. An effective-field-theory formulation of this program by Bedaque, Hammer, and van Kolck exhibits an ultraviolet RG limit cycle [5]. The proximity of physical QCD to the critical trajectory for an infrared limit cycle shows that the ultraviolet limit cycle of Ref. [5] is not just an artifact of their model.

In the late 1960's, Wilson used the renormalization group (RG) to explain universality in critical phenomena [1]. Transformations of a system that integrate out short-distance degrees of freedom while leaving the longdistances physics invariant define a $R G$ flow on the multidimensional space of coupling constants $\mathbf{g}$ for all possible terms in the Hamiltonian:

$$
\Lambda \frac{d}{d \Lambda} \mathbf{g}=\boldsymbol{\beta}(\mathbf{g}),
$$

where $\Lambda$ is an ultraviolet momentum cutoff. Standard critical phenomena are associated with infrared fixed points $\mathbf{g}_{*}$ of the RG flow, which satisfy $\boldsymbol{\beta}\left(\mathbf{g}_{*}\right)=0$. The tuning of macroscopic variables to reach a critical point corresponds to the tuning of the coupling constants $\mathbf{g}$ to a critical trajectory that flows to the fixed point $\mathbf{g}_{*}$ in the infrared limit $\Lambda \rightarrow 0$. One of the signatures of an RG fixed point is scale invariance: symmetry with respect to the coordinate transformation $\mathbf{r} \rightarrow \lambda \mathbf{r}$ for any positive number $\lambda$. This symmetry implies that dimensionless variables scale as powers of the momentum scale, perhaps with anomalous dimensions.

In a classic 1971 paper, Wilson suggested that the renormalization group might also be relevant to the strong interactions of elementary particle physics 22]. At that time, the fundamental theory for the strong interactions had not yet been discovered. It was believed to involve quarks, and hints that the strong interactions might have scaling behavior at high energies had been observed in experiments on deeply-inelastic lepton-nucleon scattering. Wilson suggested that simple high-energy behavior can be explained by simple RG flow of the relevant coupling constants in the ultraviolet limit $\Lambda \rightarrow \infty$. The simplest possibility is RG flow to an ultraviolet fixed point. Another simple possibility is RG flow to an ultraviolet limit cycle. A limit cycle is a 1-parameter family of coupling constants $\mathbf{g}_{*}(\theta)$ that is closed under the RG flow and can be parametrized by an angle $0<\theta<2 \pi$. The RG flow carries the system around a complete orbit of the limit cycle every time the ultraviolet cutoff $\Lambda$ increases by some factor $\lambda_{0}$. One of the signatures of an RG 
limit cycle is discrete scale invariance: symmetry with respect to the coordinate transformation $\mathbf{r} \rightarrow \lambda_{0}^{n} \mathbf{r}$ only for integer values of $n$. This symmetry implies that dimensionless variables with vanishing anomalous dimensions are periodic functions of the logarithm of the momentum scale with period $\ln \left(\lambda_{0}\right)$. The fundamental field theory for the strong interactions, QCD, was eventually discovered. QCD has a single coupling constant $\alpha_{s}(\Lambda)$ with an asymptotically-free ultraviolet fixed point: $\alpha_{s}(\Lambda) \rightarrow 0$ as $\Lambda \rightarrow \infty$ [6]. The renormalization of QCD does not involve an ultraviolet limit cycle.

We now turn to an independent theoretical development whose connection with RG limit cycles was first pointed out in Ref. 7]. In 1970, Efimov discovered a remarkable effect that can occur in the 3-body sector for nonrelativistic particles with a resonant short-range 2body interaction [8]. If the particles are identical bosons and if the resonance is in the $\mathrm{S}$-wave channel, the strength of the interaction is governed by the S-wave scattering length $a$. Efimov showed that if $|a|$ is much larger than the range $r_{0}$ of the interaction, there are shallow 3-body bound states whose number increases logarithmically with $|a| / r_{0}$. In the resonant limit $a \rightarrow \pm \infty$, there are infinitely many shallow 3 -body bound states with an accumulation point at the 3-body scattering threshold. The ratio of the binding energies of successive states rapidly approaches the universal constant $\lambda_{0}^{2} \approx 515$, where $\lambda_{0}=e^{\pi / s_{0}} \approx 22.7$ and $s_{0} \approx 1.00624$ is a transcendental number. In subsequent work, Efimov studied both the bound state spectrum and low-energy 3-body scattering observables for identical bosons with large scattering length [9]. He showed that the observables for different values of $a$ are related by a discrete scaling transformation in which $a \rightarrow \lambda_{0}^{n} a$, where $n$ is an integer, and lengths and energies are scaled by the appropriate powers of $\lambda_{0}^{n}$.

The Efimov effect can also occur for fermions with equal masses and large scattering lengths if they have at least 3 distinct spin states. Nucleons are examples of fermions with large scattering lengths. The spin-singlet and spin-triplet $n p$ scattering lengths are $a_{s}=-23.8 \mathrm{fm}$ and $a_{t}=5.4 \mathrm{fm}$. They are both significantly larger than the $N N$ effective range, which is $r_{0}=1.8 \mathrm{fm}$ in the spintriplet channel. Efimov used this observation as the basis for a qualitative approach to the 3-nucleon problem [4]. His starting point was to take nucleons as point particles with zero-range potentials whose strengths are adjusted to reproduce the scattering lengths $a_{s}$ and $a_{t}$. The effective range and higher order terms in the low-energy expansions of the phase shifts were treated as perturbations. This approach works well in the 2-nucleon system, giving accurate predictions for the deuteron binding energy. This is no surprise; it simply reflects the well-known success of the effective range expansion in the 2-nucleon system [10]. Remarkably, Efimov's program also works well in the 3-nucleon system at momenta small compared to $m_{\pi}$. In the triton channel, the Efimov effect makes it necessary to impose a boundary condition on the wavefunction at short distances. The boundary condition can be fixed by using either the neutron-deuteron scattering length or the triton binding energy as input. But if one of them is used as input, the other is predicted with surprising accuracy. The accuracy can be improved by taking into account the effective range as a first-order perturbation [11].

The Efimov effect was revisited by Bedaque, Hammer, and van Kolck within the framework of effective field theory (EFT) 12]. The problem of bosons with mass $m$ and large scattering length $a$ can be described by a nonrelativistic field theory with a complex-valued field $\psi$ and Hamiltonian density

$$
\mathcal{H}=\left(\hbar^{2} / 2 m\right) \nabla \psi^{*} \cdot \nabla \psi+g_{2}(\Lambda)\left(\psi^{*} \psi\right)^{2} .
$$

For convenience, we set $\hbar=1$ in the following. In the 2body sector, the nonperturbative solution of the field theory can be obtained analytically. Renormalization can be implemented by adjusting the 2-body coupling constant $g_{2}(\Lambda)$ as a function of the ultraviolet cutoff $\Lambda$ so that the scattering length is $a$. Other 2-body observables are then independent of $\Lambda$ and have the appropriate values for bosons with zero effective range.

In the 3-body sector, the nonperturbative solution of the field theory can be obtained by solving integral equations numerically. These integral equations have unique solutions only in the presence of an ultraviolet cutoff $\Lambda$. The resulting predictions for 3-body observables, although finite, depend on the ultraviolet cutoff and are periodic functions of $\ln (\Lambda)$ with period $\pi / s_{0}$. Bedaque, Hammer, and van Kolck showed that the quantum field theory could be fully renormalized to remove the residual dependence on $\Lambda$ in the 3-body sector by adding a 3-body interaction term $g_{3}(\Lambda)\left(\psi^{*} \psi\right)^{3}$ to the Hamiltonian density in (2) 12. The dependence of 3-body observables on the cutoff decreases like $1 / \Lambda^{2}$ if the 3 -body coupling constant has the form $g_{3}(\Lambda)=-4 m g_{2}(\Lambda)^{2} H(\Lambda) / \Lambda^{2}$, where the dimensionless function $H(\Lambda)$ has the form

$$
H(\Lambda)=-\frac{\sin \left[s_{0} \ln \left(\Lambda / \Lambda_{*}\right)-\arctan \left(1 / s_{0}\right)\right]}{\sin \left[s_{0} \ln \left(\Lambda / \Lambda_{*}\right)+\arctan \left(1 / s_{0}\right)\right]}
$$

for some value of $\Lambda_{*}$. With this renormalization, 3-body observables have well-defined limits as $\Lambda \rightarrow \infty$, but they depend on the parameter $\Lambda_{*}$ introduced by dimensional transmutation. Note that $H(\Lambda)$ is a periodic function of $\ln (\Lambda)$. Thus the renormalization of the field theory involves an ultraviolet limit cycle.

An effective field theory (EFT) of nucleons with contact interactions only has also been applied to the 3nucleon problem [5, 13]. This contact EFT involves an isospin doublet $N$ of Pauli fields with two independent 2-body contact interactions: $N^{\dagger} \sigma_{i} N^{c} N^{c \dagger} \sigma_{i} N$ and $N^{\dagger} \tau_{k} N^{c} N^{c \dagger} \tau_{k} N$, where $N^{c}=\sigma_{2} \tau_{2} N^{*}$. Renormalization 

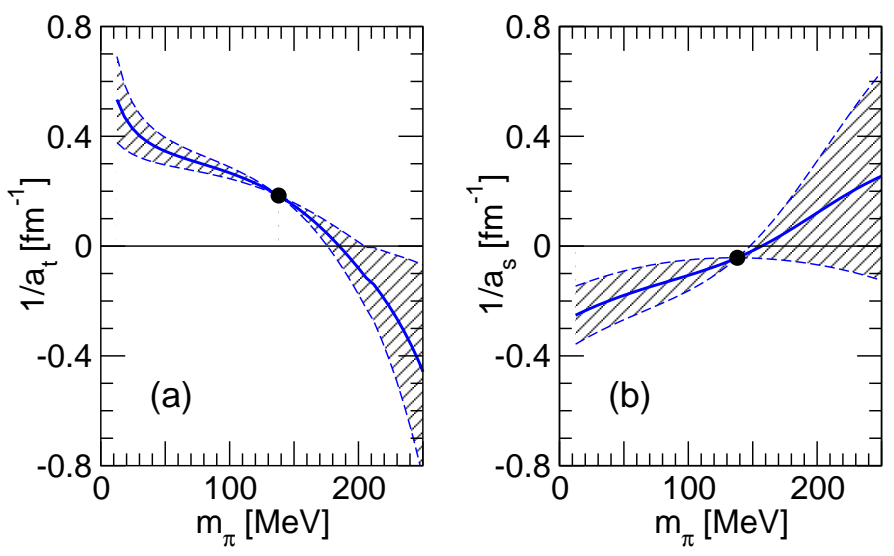

FIG. 1: The inverse scattering lengths $1 / a_{t}$ and $1 / a_{s}$ as functions of $m_{\pi}$ as predicted by the EFT with pions of Ref. [15].

in the 2-body sector requires that the two coupling constants be adjusted as a function of the ultraviolet cutoff $\Lambda$ to obtain the correct spin-singlet and spin-triplet scattering lengths $a_{s}$ and $a_{t}$. Renormalization in the 3 -body sector requires the 3-body contact interaction $N^{\dagger} \sigma_{i} N^{c} N^{c \dagger} \sigma_{j} N N^{\dagger} \sigma_{i} \sigma_{j} N$ with a coefficient proportional to (3). Thus the renormalization involves an ultraviolet limit cycle.

The low-energy few-nucleon problem can also be described by an EFT that includes explicit pion fields as well as contact interactions between the nucleons. The renormalization of such an EFT does not involve any RG limit cycle. This suggests that the RG limit cycle in the EFT of Ref. [5] is just an artifact of the model. We will argue to the contrary that it is actually a hint of an infrared limit cycle in QCD.

Our argument is based on recent work in which an EFT with explicit pions was used to extrapolate nuclear forces to the chiral limit of QCD [14, 15]. In this limit, the masses $m_{u}$ and $m_{d}$ of the up and down quarks are zero and the pion is an exactly massless Goldstone boson associated with spontaneous breaking of the chiral symmetry of QCD. According to the chiral extrapolation of Epelbaum, Meißner, and Glöckle 15], the small binding energy $2.2 \mathrm{MeV}$ of the deuteron is a fortuitous consequence of the physical values of $m_{u}$ and $m_{d}$. When extrapolated to the chiral limit, the deuteron has a much larger binding energy comparable to the scale $1 /\left(m_{N} r_{0}^{2}\right) \approx 10$ $\mathrm{MeV}$ set by the $N N$ effective range. Conversely, if extrapolated farther from the chiral limit, the deuteron's binding energy decreases to 0 and then it becomes unbound. This effect is illustrated in Fig. 1 which shows the chiral extrapolation of the inverse scattering lengths $1 / a_{t}$ and $1 / a_{s}$ as functions of $m_{\pi}$ from Ref. 15. In the EFT of Ref. [15], the coefficients of some of the 2-nucleon contact interactions are not well-constrained by the low-

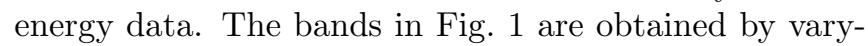
ing those coefficients over natural ranges. The width of

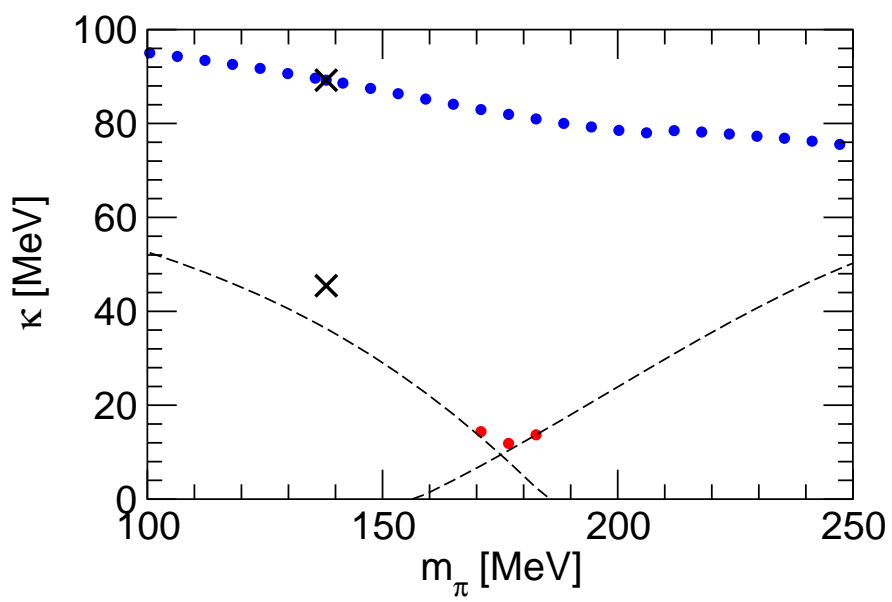

FIG. 2: The binding momenta $\kappa=\left(m B_{3}\right)^{1 / 2}$ of $p n n$ bound states as a function of $m_{\pi}$ calculated using the contact EFT of Ref. [5]. The circles indicate the triton ground state and excited state. The crosses give the binding energy of the physical deuteron and triton, while the dashed lines give the thresholds for decay into a nucleon plus a deuteron (left curve) or a spin-singlet deuteron (right curve).

the error band of course shrinks to zero at the physical value of $m_{\pi}$. The prediction of Ref. [15] is that the critical value $m_{\pi, t}$ at which $1 / a_{t}=0$ is in the range 170 $\mathrm{MeV}<m_{\pi, t}<210 \mathrm{MeV}$, which is not much larger than the physical value of $m_{\pi}$. The extrapolation of $1 / a_{s}$ has larger uncertainties. It may increase to zero at some critical value $m_{\pi, s}$ greater than $150 \mathrm{MeV}$, in which case the spin-singlet deuteron is bound for $m_{\pi}>m_{\pi . s}$, or $1 / a_{s}$ may remain negative. Beane and Savage 14] have argued that the extrapolation errors in the chiral limit are larger than estimated in Ref. [15]. For the small extrapolation to the region of larger $m_{\pi}$ where the deuteron becomes unbound, however, there is no controversy and the extrapolation errors are under control.

We now consider the chiral extrapolation of the 3-body spectrum. This could be calculated using an EFT with explicit pions. Alternatively, the chiral extrapolation can be calculated using the contact EFT of Ref. 5]. The inputs required are $a_{s}, a_{t}$, and $\Lambda_{*}$ as functions of $m_{\pi}$, which can be calculated using an EFT with pions. For the inverse scattering lengths $1 / a_{s}$ and $1 / a_{t}$, we take the central values of the error bands obtained from the chiral extrapolation in Ref. [15]. The dependence of $\Lambda_{*}$ on $m_{\pi}$ could be determined from the chiral extrapolation of the triton binding energy using an EFT with pions, but this has not yet been calculated. Like the inverse scattering lengths, $\Lambda_{*}$ should vary smoothly with $m_{\pi}$. For small extrapolations of $m_{\pi}$ from its physical value, we can approximate $\Lambda_{*}$ by a constant. We use the value $\Lambda_{*}=189$ $\mathrm{MeV}$ for $m_{\pi}=138 \mathrm{MeV}$ obtained by taking the triton binding energy as the 3-body input. In Fig. 2] we show the 3-body spectrum in the triton channel as a function of 
$m_{\pi}$. The crosses give the binding momenta $\kappa=\left(m B_{3}\right)^{1 / 2}$ of the physical deuteron and triton, while the dashed lines give the thresholds for decay into a nucleon plus a deuteron (left curve) or a spin-singlet deuteron (right curve) in the large- $a$ approximation. The circles indicate the triton ground state and excited state. In the region near $m_{\pi} \approx 175 \mathrm{MeV}$ where the decay threshold comes closest to the 3-nucleon threshold $\kappa=0$, the excited state of the triton appears in the spectrum. The existence of this very shallow excited state is a hint that the system is close to an infrared limit cycle.

If, as in the case illustrated by Fig. 2 the critical values $m_{\pi, t}$ and $m_{\pi, s}$ at which $1 / a_{t}$ and $1 / a_{s}$ go to zero satisfy $m_{\pi, s}<m_{\pi, t}$, then the deuteron and spin-singlet deuteron are both bound in the region $m_{\pi, s}<m_{\pi}<m_{\pi, t}$. Since the decay threshold never extends all the way down to the 3-nucleon threshold $\kappa=0$, there cannot be an infrared limit cycle. We argue that it is possible, however, to tune the system to the critical RG trajectory for an infrared limit cycle by adjusting the up and down quark masses $m_{u}$ and $m_{d}$. In the next-to-leading order chiral extrapolation of Ref. [15], only quark mass dependent operators proportional to $m_{u}+m_{d}$ enter. The extrapolation in $m_{\pi}$ can be interpreted as an extrapolation in the sum $m_{u}+m_{d}$, with $m_{u}-m_{d}$ held fixed. Changing $m_{u}-m_{d}$ changes the degree of isospin-symmetry breaking. Since the spin-singlet and spin-triplet channels correspond to isospin-triplet and isospin-singlet, respectively, the scattering lengths $a_{s}$ and $a_{t}$ will respond differently to changes in $m_{u}-m_{d}$. By tuning both $m_{u}$ and $m_{d}$, it should be possible to make $1 / a_{t}$ and $1 / a_{s}$ vanish simultaneously: $m_{\pi, t}=m_{\pi, s}$. This point corresponds to a critical RG trajectory for an infrared limit cycle. At this critical point, the triton has infinitely many increasinglyshallow excitations with an accumulation point at the 3 -nucleon threshold. As the excitations get more and more shallow, the ratio of the binding energies of successive states rapidly approaches $\lambda_{0}^{2} \approx 515$. Only for the deepest states like the triton will there be significant deviations. Now consider a renormalization group transformation that integrates out energies above some scale $\Lambda^{2} / m_{N}$. As $\Lambda$ is decreased, the deepest 3-nucleon bound states are removed from the spectrum, leaving only those for which the deviations from the asymptotic ratio $\lambda_{0}^{2}$ are negligible. Thus a limit cycle with a discrete-scalingsymmetry factor $\lambda_{0}$ is approached in the infrared limit $\Lambda \rightarrow 0$.

The error bands in Ref. 15] do not exclude the critical values $m_{\pi, t}$ and $m_{\pi, s}$ from satisfying $m_{\pi, t}<m_{\pi, s}$, in which case the deuteron and spin-singlet deuteron are both unbound in the region $m_{\pi, t}<m_{\pi}<m_{\pi, s}$. In this region, the decay threshold extends all the way down to the 3 -nucleon threshold $\kappa=0$. At either of the two critical values $m_{\pi, t}$ and $m_{\pi, s}$, there is a 2-nucleon bound state at threshold. However there is no Efimov effect, because the 3-body problem is not sufficiently singular at short distances. The exact infrared limit cycle can only be obtained by using an additional tuning parameter such as $m_{u}-m_{d}$ to set $m_{\pi, t}=m_{\pi, s}$.

The infrared RG limit cycle of QCD has important implications for attempts to derive nuclear physics from lattice gauge theory. The computational effort for lattice simulations increases dramatically as the pion mass decreases and is prohibitive at the physical value. Lattice simulations are typically carried out at a value of $m_{\pi}$ that is 2-3 times larger than the physical value, and then a chiral extrapolation is made to to $m_{\pi}=138 \mathrm{MeV}$. Unfortunately, this requires extrapolating past the region of $m_{\pi}$ where there is an RG limit cycle, which may introduce large extrapolation errors. The proximity of physical QCD to the critical trajectory for the infrared limit cycle explains the success of Efimov's program [4] for describing the 3-nucleon problem using zero-range forces or a contact EFT [5]. The apparent convergence of the effective-range corrections for momenta of order $m_{\pi}$ [13] can be explained if the momentum expansion in the contact EFT is in powers of $p / m_{\pi}^{*}$, where $m_{\pi}^{*} \approx 175 \mathrm{MeV}$ is the critical value of the pion mass, which is significantly larger than the physical value. Our final conclusion is that the ultraviolet limit cycle in the contact EFT [5] is not just an artifact of the model, but a hint of the existence of the infrared limit cycle of QCD.

This research was supported in part by DOE grant DE-FG02-91-ER4069. We thank U.-G. Meißner for useful discussions, E. Epelbaum for providing the scattering lengths $a_{s}$ and $a_{t}$ as functions of $m_{\pi}$, and R.J. Furnstahl for comments on the manuscript.

[1] K. G. Wilson, Rev. Mod. Phys. 55, 583 (1983).

[2] K. G. Wilson, Phys. Rev. D 3, 1818 (1971).

[3] S. D. Glazek and K. G. Wilson, Phys. Rev. Lett. 89, 230401 (2002).

[4] V. Efimov, Nucl. Phys. A 362, 45 (1981).

[5] P. F. Bedaque, H.-W. Hammer, and U. van Kolck, Nucl. Phys. A 676, 357 (2000).

[6] D. J. Gross and F. Wilczek, Phys. Rev. Lett. 30, 1343 (1973); H. D. Politzer, Phys. Rev. Lett. 30, 1346 (1973).

[7] S. Albeverio, R. Hoegh-Krohn, and T. T. Wu, Phys. Lett. A 83, 105 (1981).

[8] V. N. Efimov, Sov. J. Nucl. Phys. 12, 589 (1971).

[9] V. N. Efimov, Sov. J. Nucl. Phys. 29, 546 (1979).

[10] H. A. Bethe, Phys. Rev. 76, 38 (1949).

[11] V. Efimov, Phys. Rev. C 44, 2303 (1991).

[12] P. F. Bedaque, H.-W. Hammer, and U. van Kolck, Phys. Rev. Lett. 82, 463 (1999); Nucl. Phys. A 646, 444 (1999).

[13] H.-W. Hammer and T. Mehen, Phys. Lett. B 516, 353 (2001); P. F. Bedaque, G. Rupak, H. W. Griesshammer, and H.-W. Hammer, Nucl. Phys. A 714, 589 (2003).

[14] S. R. Beane and M. J. Savage, Nucl. Phys. A 717, 91 (2003); 713, 148 (2003).

[15] E. Epelbaum, U.-G. Meißner, and W. Glöckle, Nucl. Phys. A 714, 535 (2003). 